Center for

Mathematical Economics

Working Papers

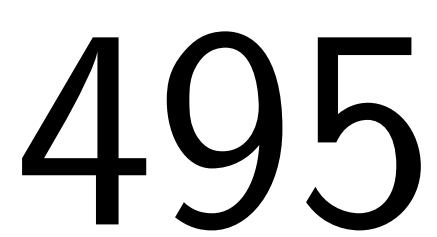

December 2013

\title{
Welfare Improving discrimination based on Cognitive Limitations
}

Oktay Sürücü

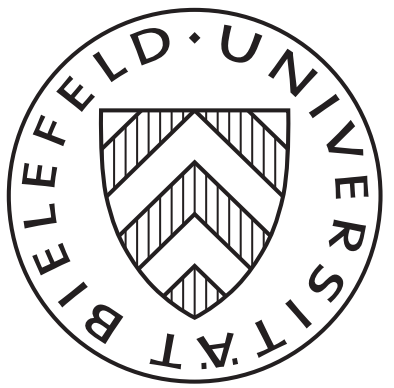




\title{
Welfare Improving Discrimination based on Cognitive Limitations
}

\author{
OKTAY SÜRÜCÜ \\ Center for Mathematical Economics, Universität Bielefeld, \\ oktay.surucu@uni-bielefeld.de
}

December, 2013

\begin{abstract}
This paper is concerned with the situation in which a profit-maximizing monopolist faces consumers that are diverse not only in their preferences but also in their levels of bounded rationality. The behavioral phenomenon considered here is the attraction effect when choices are made across categories. Using the standard second-degree price discrimination model, the optimal menu of contracts that screens consumers' types is characterized. The benefit of discriminating consumers based on their preference and cognitive limitation is always higher than its cost. In other words, the monopolist can exploit consumers and increase his profit with this contract. The model provides a possible explanation for the apparent puzzle why one may observe that the same quality products are priced differently under different labels. Moreover, this contract is welfare improving.
\end{abstract}

Keywords: bounded rationality, attraction effect, contract design, welfare.

JEL Classification Numbers: D03, D42, D60, D82, D86. 


\section{Introduction}

The growing literature initiated by Amos Tversky, Daniel Kahneman, and their collaborators presents a large body of experimental evidence demonstrating that human beings depart systematically from full rationality due to their cognitive imperfections. This emerged a literature strand that integrates insights gained from experimental studies into economical models. This paper contributes to this body of work by exploring the impact of cognitive limitations on mechanism design. More specifically, it is concerned with the attraction effect which refers to a phenomenon where adding a third alternative that is inferior to only one of the two alternatives affects the choice. This phenomenon is first demonstrated by Huber, Payne and Puto (1982) and then corroborated not only in field experiments but also in numerous laboratory experiments both in hypothetical and real choices. The main focus of this paper is on this effect when choices are made between alternatives across categories. As an illustration, consider the following experiment conducted by Simonson and Tversky (1992). There are two versions in this experiment and in each version subjects are asked to choose one item out of three alternatives. In the first version, these alternatives are two different brands of towel, one of which is of high and the other of low quality, and a high quality brand of tissue. In the second version, low quality brand of towel is replaced by a low quality brand of tissue. That is, the two decision sets differ only in the low quality item they contain. These low quality products are inferior options since they are presented together with their high quality brands. Under the assumption of full rationality, inferior options are irrelevant and therefore these two sets of alternatives are equivalent. However, the results show that the market share of high quality brand of towel in the first version is significantly greater than its share in the second version. This shows that even though low quality items are almost never chosen, they are not irrelevant; their existence affects choices. That is, the decisions made in this experiment are context-dependent. The results further show that a significant number of subjects tend to choose the high quality item which is presented together with its low quality alternative. This suggests that introducing an inferior option in a category may help to increase the attractiveness of that category. The above fact may be a possible explanation why shopkeepers prefer to put fancy and highly expensive or very cheap but low quality products on their shop windows. Even though it is highly unlikely for these products to be sold, they attract attention to the shop and thus increase the probability of sales. This type of inferior products are referred to as decoys or attention grabbers in literature, since they attract the attention of individuals not on themselves but on the category to which they belong. Agents who are affected by these products are called context-biased in this paper.

The following example, inspired by Eliaz and Spiegler (2010), illustrates a possible usage of attention grabbers. A consumer who wants to buy a new cellular phone may notice that a store offers a model that is very small in size. In order to inspect this 
model, he enters the store and finds out that other features of this model are not that good (e.g., it does not have a camera). Being already in the store, the consumer may browse other models and find one that suits his needs. Thus, even though the phone in display is not sold, it grabs the attention of consumers and directs them to the store. However, it would be implausible to expect the same reaction from every consumer. One might be very naïve and constantly fall for the attraction of a decoy and therefore be gravitated towards it, but there might be others who are more sophisticated such that they never or seldom fall for attraction. That is, agents' cognitive limitations might differ and they may be diversely context-biased.

Integrating this type of behavior into mechanism design, I study the problem of a principal who faces agents differing both in preferences and in the level of context-bias. That is, an agent's type is a two dimensional object. The first dimension corresponds to her valuation for quality and it is a binary variable taking values either high or low. The second dimension stands for her level of context-bias and is a continuous variable. The principal cannot observe the types and therefore wants to design a mechanism that screens and discriminates them in order to increase his profit. I use the representation of choices proposed in Barbos (2010) and integrate it into the standard second-degree price discrimination model to characterize the optimal contract. This axiomatized and essentially unique representation includes a variable $\theta \in[0,1]$ that captures individuals' level of context bias. An agent with $\theta=0$ is not affected by any attention grabber and therefore, she is fully rational. As $\theta$ increases agents' level of context-bias increases thus the degree of sophistication decreases. The highest affection is shown by fully naïve agents for whom $\theta=1$.

Agents make their choices as if they first evaluate all exogenously given categories and then choose the best option that belongs to the category they value most. Cognitive imperfections step in only when agents evaluate categories. A fully rational agent considers only her most preferred alternative, whereas a naïve agent may get distracted by an attention grabber and fail to evaluate categories normatively. Once an agent concentrates on the category that she values most, she choses the normatively best option from that category. In other words, cognitive limitations may lead an agent to choose the best option from a not-the-best category.

As a motivating example, consider a manufacturer who wants to screen consumers' type and discriminate among them. He needs to generate different categories, each of which attracts different types. This can be achieved by creating different brands 1 If two products are of the same brand consumers perceive them as belonging to the same category, otherwise they are mostly perceived as being from different categories. Furthermore, careful usage of attention grabbers under a brand label would allow the

1 Procter \& Gamble and Tod's Group are two examples of companies that produce the same product under different brand names. Tide, Ariel, Gain and Dash are some of the detergent brands owned by Procter \& Gamble and Tod's and Hogan are the two shoe brands of Tod's Group. 
manufacturer to direct the attention of a specific type to that brand. For example, he can create a luxury or a premium brand for consumers who easily fall for attraction and charge a very high price.

In conventional price discrimination models, all the surplus is extracted from low valuation agents and high valuation agents are given informational rents in order to prevent them from mimicking low valuation agents. However, I show that the principal can increase his profit by designing a mechanism that screens high valuation agents' degree of sophistication and exploits their bounded rationality. The optimal contract has three menus. The first one is designed for low valuation agents and, as in conventional models, extracts their entire surplus. The other two menus partition the space of high valuation consumer types into two subsets based on a threshold level for $\theta$. Due to their sophistication, the principal cannot extract much from high valuation agents whose $\theta$ is lower than the threshold. That is, relatively sophisticated high valuation types' bounded rationality is not exploited and therefore, they receive full informational rents. The principal makes use of attention grabbers and exploits the rest of high valuation types. These types receive only partial informational rents. A very interesting characteristic of the contract is that it offers the same quality product in both menus designed for high valuation types but with different prices. That is, naïve agents end up paying more for the same quality product sold to more sophisticated types. Furthermore, the quality of the product sold to low valuation types is higher than it is in a conventional price discrimination model, and is closer to its value under the perfect information case.

The results suggest that the manufacturer in the motivating example should create three brands. The first one is for low valuation consumers, the products are cheap and of low quality. The second brand is for relatively sophisticated high valuation consumers and under this brand high quality products are sold with reasonable prices. Finally, the third brand is for naïve high valuation consumers. The products of this brand have the same quality as the ones sold under the second brand, but they are highly expensive. This observation provides a possible explanation to the apparent puzzle why one may observe products of the same quality sold at different prices under different labels. Volkwagen Group, for example, has many different brands including Audi, Seat, Škoda and Volkswagen. Even though the price vary considerable, the Group is said to use the same platforms and engines, and even the same body, for a wide range of cars across its brands $2^{2}$

Finally, taking the standard price discrimination model as benchmark, I discuss the welfare differences under the optimal contract in consideration. The principal is strictly better off since he can exploit agents' bounded rationality with the present contract. For low valuation types there is no difference: their entire surplus is extracted and

\footnotetext{
${ }^{2}$ http://www.autoexpress.co.uk/
} 
they end up with zero utility in both models? However, the quality of the product sold to these types is higher than it is in the benchmark and therefore, is closer to its value under perfect information case. As a consequence of this result, the utility of sophisticated high valuation types is higher in the present model, since they receive full informational rents. The welfare difference for naïve high valuation types is more subtle. While the product designed for these types has the same quality in both models, the effect of discriminating agents based on their cognitive limitation on the price of this product is not straightforward. This is due to the fact that there are two opposing forces. The first one decreases the price due to the same informational rents argument as for sophisticated types, whereas the second one increases it owing to the fact that these types' bounded rationality is exploited. With the assumptions of this paper, it is not possible to say anything about the net effect on the price since it requires further specifications on the relationship between the distribution function of $\theta$ for high valuation types and agents' utilities. The welfare difference on aggregate level instead can be determined clearly without any further specifications. The increase in high valuation types' welfare is due to the increase in their informational rents which is paid by the principal. Likewise, the change in naïve valuation types' welfare is reflected on the profit so that on aggregate level they cancel each other out. However, this is not the case with low valuation types. The quality of the product designed for these types is closer to its value under the full information case, therefore the principal's profit obtained from low valuation types is higher. However, this benefit of the principal is not reflected on these types because they end up with the same level of utility in both models. This implies that discriminating agents based on their cognitive limitation improves the welfare on aggregate level.

\section{Related Literature}

The representation of choices used in this paper is borrowed from Barbos (2010). This representation is axiomatized and shown to be essentially unique. Furthermore, Barbos presents an application of this representation to principal-agent models. In this application, however, agents are homogeneous with respect to the level of cognitive limitations. In other words, there are only two types in the market and they differ only in preferences for quality, but not in their cognitive limitations. Furthermore, the principal knows agents' level of context-bias. In contract, in the present paper, agents are diversely context-biased and types are private information. As a result of the introduction of heterogeneity in agents' type, the characterization of the optimal contract changes dramatically. A distinguishing feature of this contract is that it provides enlightenment on the puzzle why one may observe products of the same quality sold at different prices under different labels.

\footnotetext{
${ }^{3}$ Outside options in both cases are assumed to be zero.
} 
This paper belongs to the small literature on contracting with diversely bounded rational agents. The idea of screening consumer types and discriminating them based on their level of cognitive imperfections first appears in Rubinstein (1993). In this paper, consumers differ in their ability to process information regarding the economic market. It is shown that by complicating the price offers, a monopolist can limit the number of consumers accepting his offer. A crucial assumption made in this paper is that the high type (the type with low cost of production) is more sophisticated than the low type, i.e., the high type is more capable of processing information than the low type. This assumption narrows down the real life situations that are covered by Rubinstein's model. Piccione and Rubinstein (2003) model cognitive differences among agents using the concept of DeBruijin sequences. They show that in a setting where consumers have diverse ability to grasp market behavior, price fluctuations that are independent of economic fundamentals can emerge in equilibrium. Furthermore, this can be recognized only by more sophisticated agents.

One of the most closely related works to the present one is Eliaz and Spiegler (2006). They consider a market in which there is a continuum of types differing in their level of cognitive limitations and the principal discriminates between types. The main difference between the present work and Eliaz and Spiegler (2006) is the type of bounded rationality. I consider agents who are diversely context-biased, whereas in Eliaz and Spiegler (2006) agents have diverse cognitive abilities to forecast changes in their future tastes. Even though the sources of bounded rationality are different, the way the principal makes use of it is very similar. In Eliaz and Spiegler (2006), a fully naïve agent does not anticipate that her utility function will change in the second period and therefore, is attracted by a contract that allows her to take the optimal action based on her first period utility function (imaginary action) even if the optimal action based on her second period utility function (real action) is extremely costly under this contract. An imaginary action serves as an attention grabber and gravitates naïve agents towards a contract that exploits them by providing a highly expensive real action. Similarly, in the present paper, the principal uses an inferior product an as attention grabber which directs naïve agents to a contract that exploits them by using a highly expensive superior product. The optimal contract in Eliaz and Spiegler (2006) has some similar features with the optimal contract characterized in the present paper. There exists a threshold that partitions the type space into two subspaces as (i) relatively sophisticated types who are assigned a contract that serves as a perfect commitment device and therefore, they are not exploited and (ii) relatively naïve types whose bounded rationality is exploited. In the present work, however, types do not differ only in the level of bounded rationality but also in preferences. Therefore, the optimal contract has an additional menu designed for low valuation types on top of the two menus having similar features as the ones in Eliaz and Spiegler (2006). 


\section{The Model}

I study a second-degree price discrimination model in which a principal faces a continuum of agents differing in their preference and level of context bias. In conventional price discrimination models, informational rents are given to high types in order to give them incentive not to mimic low types. This lowers the profit of the principal when compared to the perfect information case. In this model, I consider a principal who takes agents' cognitive limitations into consideration while maximizing his expected profit. Differently put, the principal is after exploiting agents' bounded rationality.

Products on the market are characterized by quality-price pair, $(q, p) \in \mathbb{R}_{+}^{2}$. Agents differ in their tastes for quality; $\lambda \in(0,1)$ share of them have relatively lower valuation for quality than the rest of the agents, and these valuations are denoted by $v_{l}($.$) and$ $v_{h}($.$) for low and high valuation types, respectively. The utility of an agent is quasilinear$ in price and is given by

$$
u_{i}(q, p)=v_{i}(q)-p,
$$

where $i \in\{h, l\}$. The single crossing property is satisfied $\left(v_{h}^{\prime}(q)>v_{l}^{\prime}(q)\right.$, for all $\left.q \in \mathbb{R}_{+}\right)$ and without loss of generality the outside option for agents is assumed to be zero.

I use the reference-dependent representation, axiomatized by Barbos (2010), for agents' choices. It is consistent with the earlier mentioned experimental evidence presented by Simonson and Tversky (1992). According to this representation, an agent makes her decision as if she first compares her subjective valuations of the exogenously given categories, and then chooses the objectively best product from the category which she values most. An agent's valuation for a finite category $A$ is given by

$$
V(A, \theta)=\max _{\{x \in A \mid u(x) \geq 0\}} u(x)-\theta \min _{\{y \in A \mid u(y) \geq 0\}} u(y),
$$

and is subjective since it depends on the agent's level of context bias, which is denoted by $\theta \in[0,1]$. The choice of product from the category that an agent values most is determined by

$$
c(A)=\arg \max _{x \in A} u(x) .
$$

Being context-biased affects only the decision regarding the choice of the category. This comes from to the fact that the presence of an inferior option (attention grabber) in a category may increase the attractiveness of that category, as suggested by the experiment from Simonson and Tversky (1992). When $\theta$ takes its lowest possible value zero, the second term of equation (1) disappears and in this case, the agent's valuation for a finite category depends only on the alternative that provides the highest utility. In other words, type $\theta=0$ does not take inferior options into account and therefore is not context biased (i.e., fully rational). For an agent with a strictly positive $\theta$, the resulting choice of category may be different, since this time the valuations are affected by inferior options. That is to say, a non-zero $\theta$ type is more naïve than 0 -type owing to 
the fact that this type might be gravitated towards a category by the attention grabber of that category. Furthermore, the formulation in (1) considers only alternatives that provide non-negative utilities, i.e., alternatives that yield higher utility than the outside option. This means that when evaluating a category, agents examine products that they might end up buying. When all items in a category provide an agent with negative utility, it is assumed that her valuation for this category is zero.

The following example illustrates how the decision process works. Consider the first version of the experiment from Simonson and Tversky (1992). Subjects are asked to choose between a high quality brand of tissue, a high quality brand of towel and a low quality brand of towel denoted by $y, x$ and $x^{\prime}$, respectively. Assume that the utilities of the alternatives are $u(y)=12, u(x)=10$ and $u\left(x^{\prime}\right)=5$. Then the valuations of the categories for an agent with $\theta=0.5$ are

$$
\begin{aligned}
V(\text { Tissues, } \theta=0.5) & =u(y)-\theta u(y) \\
& =12-0.5 \times 12=6 \\
V(\text { Towels, } \theta=0.5) & =u(x)-\theta u\left(x^{\prime}\right) \\
& =10-0.5 \times 5=7.5
\end{aligned}
$$

Since towels category has a higher subjective value to this agent, she focuses on this category and chooses the high quality towel $x$. In this example, we see that even though the high quality brand of tissue $y$ provides the agent with the highest utility, she ends up choosing the high quality brand of towel $x$. Observe that types with $\theta<\frac{2}{7}$ make the optimal choice since they are sophisticated enough not to be fooled by the attractiveness of the towel category.

\section{The Optimal Contract}

In this section, I characterize the optimal menu of contracts when a principal faces a continuum of agents differing in their preference for quality and level of context bias. More specifically, an agent's type is a two dimensional object that consists of her valuation for quality and her level of context-bias. The first entry is a binary variable which is either high or low, whereas the second one, denoted by $\theta$, takes values in a continuum $\theta \in[0,1]$. In order to maximize his profit, the principal wants to discriminate agents based on their types. Products are characterized by quality-price pair, and a product designed for a type whose valuation for quality is $i \in\{l, h\}$ and level of context bias is $\theta$ is denoted by $\left(q_{i}(\theta), p_{i}(\theta)\right) \in \mathbb{R}_{+}^{2}$. Furthermore, each menu of the contract consists of a primary and a secondary product. A secondary product serves as an attention grabber. That is, it attracts attention of a specific type on the menu to which it belongs. A type who is gravitated towards it buys the primary product of the menu since it provides higher utility than the secondary product. The notation used for a secondary product designed for a $(i, \theta)$ type is $\left(q_{i}^{\prime}(\theta), p_{i}^{\prime}(\theta)\right) \in \mathbb{R}_{+}^{2}$. 
The optimal contract can be characterized by the following optimization problem:

$$
\max _{\left\{\left(q_{i}(\theta), p_{i}(\theta)\right),\left(q_{i}^{\prime}(\theta), p_{i}^{\prime}(\theta)\right)\right\}} \lambda \int_{0}^{1}\left[p_{l}(\theta)-c\left(q_{l}(\theta)\right)\right] \mathrm{d} G(\theta)+(1-\lambda) \int_{0}^{1}\left[p_{h}(\theta)-c\left(q_{h}(\theta)\right)\right] \mathrm{d} F(\theta)
$$

subject to, for $i, j \in\{h, l\}$ and $\forall \phi \in[0,1]$

$$
\begin{aligned}
& u_{i}\left(q_{i}^{\prime}(\theta), p_{i}^{\prime}(\theta)\right) \geq 0 \\
& u_{i}\left(q_{i}(\theta), p_{i}(\theta)\right) \geq u_{i}\left(q_{i}^{\prime}(\theta), p_{i}^{\prime}(\theta)\right) \\
& u_{i}\left(q_{i}(\theta), p_{i}(\theta)\right)-\theta u_{i}\left(q_{i}^{\prime}(\theta), p_{i}^{\prime}(\theta)\right) \geq u_{i}\left(q_{j}(\phi), p_{j}(\phi)\right)-\theta u_{i}\left(q_{j}^{\prime}(\phi), p_{j}^{\prime}(\phi)\right)
\end{aligned}
$$

where $\lambda \in(0,1)$ is the share of agents whose valuation for quality is low, $G(\theta)$ and $F(\theta)$ are the continuous distribution functions of $\theta$ for low and high valuation agents, respectively. Finally, $c($.$) is the principal's cost function, that is assumed to be increasing,$ convex and differentiable.

The first constraint ensures that secondary products have the potential to attract attention of the types for whom they are designed. This constraint is needed since agents consider only the products they might end buying when evaluating a category. The second constraint says that each primary product provides higher utility than the secondary product of that menu. This guarantees that an agent who is gravitated towards a menu by its attention grabber, chooses the primary product of that menu. Finally, the third one is the incentive compatibility constraint ensuring that no type is better off by mimicking another type.

The first result shows that the optimal contract inherits one of the main features of conventional price discrimination models.

Proposition 1. The optimal contract contains only a single menu designed for low valuation types and it extracts the entire surplus from these types.

As in any conventional price discrimination model, the principal can discriminate between high and low valuation types and extract the entire surplus from low valuation types. This result comes from the fact that the single crossing property holds. The discrimination of low valuation types from high valuation types is not achieved through their cognitive skills but through their preferences, that is, by using the fact that they differ in valuation for quality. Therefore, the distribution of $\theta$ for low valuation types $G(\theta)$ is of no importance to the principal.

The surplus from high valuation types, however, cannot be extracted entirely. They are given some informational rents in order to prevent them from choosing the menu designed for low valuation types. The optimal contract minimizes these informational rents making use of context-bias. Therefore the types, whose bounded rationality is exploited, if any, are among high valuation types. 
For reasons of convenience, I make the following adjustments in notations. First, I suppress subscripts for high valuation types. Second, I fill the room created by the first adjustment with $\theta$. For example, from now on, the notation $u\left(q_{\theta}, p_{\theta}\right)$ stands for the utility of a high valuation agent from the product designed for a high valuation $\theta$ type. Finally, since the level of context bias of low valuation types are of no importance, I drop $\theta$ from the products designed for these types and denote their utility that they receive from these products as $u_{l}\left(q_{l}, p_{l}\right)$.

Having seen the main characteristic of the menu designed for low valuation types, let us turn our attention to other menus of the contract. The following proposition provides the first result related to high valuation types and bears out the intuition that more naïve types are more heavily exploited.

Proposition 2. In the optimal contract, the utility provided by primary and secondary products for high valuation types $\left(u\left(q_{\theta}, p_{\theta}\right)\right.$ and $\left.u\left(q_{\theta}^{\prime}, p_{\theta}^{\prime}\right)\right)$ are decreasing in $\theta$.

The intuition for this result is as follows. As $\theta$ increases, the level of context bias increases. Thus, an agent with a high $\theta$ falls easily for attraction. The principal uses this fact and creates an attractive menu for high $\theta$ types that exploits their bounded rationality. Agents' subjective valuation for a menu, given in equation (1), is increasing as the utility of the secondary product decreases. Therefore, in order to make a menu look attractive to a naive eye, the principal sets a low utility level for its secondary product. The fact that this attractive menu is an exploitative one implies that it's primary product provides agents with a low utility as well.

An implication of this monotonicity result is the following:

Corollary 1. Suppose that a primary product of a menu is chosen by two different high valuation types, say $\theta_{1}$ and $\theta_{2}$ such that $\theta_{1}<\theta_{2}$. Then any high valuation type with $\theta \in\left(\theta_{1}, \theta_{2}\right)$ chooses the same product.

This result implies that the optimal contract partitions the type space into blocks of connected intervals such that only one menu is designed for all the types belonging to the same block. Furthermore, Proposition 2 together with Corollary 1 yields the following observation:

Observation 1. The secondary product of the menu designed for the group of types, among which is the most naïve high valuation type $(\theta=1)$, provides zero utility.

This observation is a natural consequence of the fact that as the utility provided by a secondary product decreases, the attractiveness of the menu to which it belongs increases. In order to benefit the most from bounded rationality, the optimal contract offers highly naïve types a menu that is highly attractive.

On the other extreme is the block of the type partition, among which is the fully rational high valuation type $(\theta=0)$. The fully rational type is not affected by the 
attractiveness of secondary products and considers only primary products in her valuation of exogenously given menus. In other words, this type cannot be exploited and receives full informational rents.

Proposition 3. There exists a threshold $\bar{\theta} \in(0,1]$, such that for every high valuation type with $\theta \leq \bar{\theta}$

$$
u\left(q_{\theta}, p_{\theta}\right)=u\left(q_{\theta}^{\prime}, p_{\theta}^{\prime}\right)=u\left(q_{l}, p_{l}\right)=u\left(q_{l}^{\prime}, p_{l}^{\prime}\right) .
$$

The intuition behind this result is as follows. High valuation types whose level of context bias is below the threshold are so sophisticated that they act almost rationally and they almost never fall for attraction. Thus these types cannot be exploited and are given full informational rents. However, the principal wants to exploit the rest of the high valuation types and therefore, does not want them to choose this nonexploitative menu. In order to make it look unattractive, he sets the utility of the secondary product to its highest possible value. This explains the first equality in Proposition 3. The other two equalities follow from the fact that since the menu designed for sophisticated high valuation types provides full informational rents, their incentive compatibility constraint binds for the menu designed for low valuation types.

Proposition 3 implies that the set of exploited types consists of high valuation agents whose context bias level is greater than the threshold. This set might be empty if the threshold $\bar{\theta}$ turns out to be 1 . This means that, when the threshold is 1 , the present model yields exactly the same contract as in any conventional price discrimination models, where none of the agents' bounded rationality is exploited and the discrimination is based only on preferences for quality.

Before proceeding with the menus designed for naïve high valuation types, let us turn our attention to incentive compatibility constraints. Let $V(\theta)$ denote the subjective valuation of a high valuation $\theta$ type for the menu designed for her type, that is

$$
V(\theta) \equiv u\left(q_{\theta}, p_{\theta}\right)-\theta u\left(q_{\theta}^{\prime}, p_{\theta}^{\prime}\right)
$$

The following result is obtained by following Krishna's derivation of incentive compatibility for direct mechanisms.

Proposition 4. The incentive compatibility constraint for all high valuation types implies

$$
V(\theta)=u\left(q_{0}, p_{0}\right)-\int_{0}^{\theta} u\left(q_{x}^{\prime}, p_{x}^{\prime}\right) \mathrm{d} x
$$

Using equations (3) and (4), the difference between the utilities provided by a primary and a secondary product can be written as follows:

$$
u\left(q_{\theta}, p_{\theta}\right)-u\left(q_{\theta}^{\prime}, p_{\theta}^{\prime}\right)=\theta u\left(q_{0}, p_{0}\right)-\int_{0}^{\theta} u\left(q_{x}^{\prime}, p_{x}^{\prime}\right) \mathrm{d} x+(1-\theta)\left[u\left(q_{0}, p_{0}\right)-u\left(q_{\theta}^{\prime}, p_{\theta}^{\prime}\right)\right] .
$$


This difference is positive since $u\left(q_{0}, p_{0}\right)$ can be replaced by $u\left(q_{0}^{\prime}, p_{0}^{\prime}\right)$ and $u\left(q_{\theta}^{\prime}, p_{\theta}^{\prime}\right)$ is decreasing in $\theta$ according to Proposition 2 and Proposition 3 . That is, the equation for $V(\theta)$ given in (4) implies that for a high valuation type, the utility difference between a primary and a secondary product of the menu designed for her is non-negative. Therefore, replacing the incentive compatibility constraint with the representation $V(\theta)$, given in (4), guarantees that the second constraint of the optimization problem (2.2) is satisfied.

As a last step before reformulating the principal's problem, I derive the expression for the price of the primary product designed for high valuation $\theta$ type. Substituting the definitions for $V(\theta)$ and $u\left(q_{\theta}, p_{\theta}\right)$ into equation (4) yields:

$$
p_{\theta}=v\left(q_{\theta}\right)-\theta u\left(q_{\theta}^{\prime}, p_{\theta}^{\prime}\right)-u\left(q_{0}, p_{0}\right)+\int_{0}^{\theta} u\left(q_{x}^{\prime}, p_{x}^{\prime}\right) \mathrm{d} x .
$$

The fact that there is only one menu designed for high valuation types below the threshold implies $\left(q_{\theta}, p_{\theta}\right)=\left(q_{0}, p_{0}\right),\left(q_{\theta}^{\prime}, p_{\theta}^{\prime}\right)=\left(q_{0}^{\prime}, p_{0}^{\prime}\right)$ for $\theta \leq \bar{\theta}$. Furthermore, the utility of a high valuation type that she gets from the product designed for low valuation types is given as $u\left(q_{l}, p_{l}\right)=v\left(q_{l}\right)-p_{l}=v\left(q_{l}\right)-v_{l}\left(q_{l}\right)$. The first equality comes from the definition of utility assumed in the present paper and the second one from the fact that this product extracts the entire surplus from a low valuation type. Now, the above expression for $p_{\theta}$ can be re-written as a piecewise function.

$$
p_{\theta}= \begin{cases}v\left(q_{0}\right)-v\left(q_{l}\right)+v_{l}\left(q_{l}\right) & \text { if } \theta \leq \bar{\theta} \\ v\left(q_{\theta}\right)-\theta u\left(q_{\theta}^{\prime}, p_{\theta}^{\prime}\right)-(1-\bar{\theta}) u\left(q_{0}, p_{0}\right)+\int_{\bar{\theta}}^{\theta} u\left(q_{x}^{\prime}, p_{x}^{\prime}\right) \mathrm{d} x & \text { if } \theta>\bar{\theta}\end{cases}
$$

Using all the results obtained so far, principal's optimization problem can be restated as follows:

$$
\begin{aligned}
& \max _{\left\{q_{l}, q_{0}, q_{\theta},\left(q_{\theta}^{\prime}, p_{\theta}^{\prime}\right)\right\}} \lambda\left[v_{l}\left(q_{l}\right)-c\left(q_{l}\right)\right]+(1-\lambda) F(\bar{\theta})\left[v\left(q_{0}\right)-v\left(q_{l}\right)+v_{l}\left(q_{l}\right)-c\left(q_{0}\right)\right] \\
& \quad+(1-\lambda) \int_{\bar{\theta}}^{1}\left[v\left(q_{\theta}\right)-c\left(q_{\theta}\right)-\theta u\left(q_{\theta}^{\prime}, p_{\theta}^{\prime}\right)-(1-\bar{\theta})\left(v\left(q_{l}\right)-v_{l}\left(q_{l}\right)\right)+\int_{\bar{\theta}}^{\theta} u\left(q_{x}^{\prime}, p_{x}^{\prime}\right) \mathrm{d} x\right] \mathrm{d} F(\theta)
\end{aligned}
$$

subject to $u\left(q_{\theta}^{\prime}, p_{\theta}^{\prime}\right) \geq 0$.

Remark 1. The last term of the objective function that captures principal's profit from high valuation agents whose $\theta>\bar{\theta}$ can be written as:

$$
(1-\lambda) \int_{\bar{\theta}}^{1}\left[v\left(q_{\theta}\right)-c\left(q_{\theta}\right)-(1-\bar{\theta})\left(v\left(q_{l}\right)-v_{l}\left(q_{l}\right)\right)-\left(v\left(q_{\theta}^{\prime}\right)-p_{\theta}^{\prime}\right) \Phi(\theta)\right] f(\theta) \mathrm{d} \theta
$$


where $\Phi(\theta)=\theta-\frac{1-F(\theta)}{f(\theta)}$.

Here, I assume that the hazard rate $f(\theta) /(1-F(\theta))$ is a continuous and increasing function. This is a standard assumption made in mechanism-design literature and implies that $\Phi(\theta)$ is also increasing. Under this assumption, the following result that characterizes the optimal menu designed for high valuation types with $\theta>\bar{\theta}$ is obtained.

Proposition 5. The primary and secondary products designed for high valuation agents for whom $\theta>\bar{\theta}$ are determined as follows:

$$
\begin{aligned}
v^{\prime}\left(q_{\theta}\right) & =c^{\prime}\left(q_{\theta}\right) \\
v\left(q_{\theta}^{\prime}\right) & =p_{\theta}^{\prime}
\end{aligned}
$$

The quality of the primary product is the same as in the perfect information case, that is, the marginal utility is equal to the marginal cost. The secondary product instead provides high valuation types with zero utility since its price is exactly equal to how much a high valuation type is willing to pay. Since the quality of the secondary product does not enter into the objective function, the principal could choose any quality level for this product as long as it is priced according to Equation 6. This implies that the attention grabber could be a very high quality but expensive product as well as a very cheap but of low quality product. Since the attractiveness of a menu increases as the utility provided by its attention grabber decreases, this menu is created in such a way that it looks fetching to the max in order to attract naïve types. Moreover, the fact that neither the quality nor the price of the products of this menu depend on $\theta$ yields the following important remark:

Remark 2. There exists only one menu designed for high valuation agents with $\theta>\bar{\theta}$.

This remark implies that the optimal contract has three menus partitioning the type space into three subsets. Low valuation types choose the menu that extracts their entire surplus. The menu designed for relatively sophisticated high valuation types does not exploit their bounded rationality and give them full informational rents. From now on, the products of this menu will be denoted by the subscript 0 indicating the level of context-bias of the most sophisticated type for whom this menu is designed. That is, $\left(q_{0}, p_{0}\right)$ and $\left(q_{0}^{\prime}, p_{0}^{\prime}\right)$ denote the primary and the secondary product of this menu, respectively. Finally, relatively naïve high valuation types fall for the attraction of the menu that exploits their bounded rationality and thus decreases their informational rents. Similarly, $\left(q_{\bar{\theta}}, p_{\bar{\theta}}\right)$ and $\left(q_{\bar{\theta}}^{\prime}, p_{\bar{\theta}}^{\prime}\right)$ denote the products of this menu.

As seen in the proof of this Proposition 5 (see Appendix), the optimal threshold $\bar{\theta}$ is the zero of the function $\Phi(\theta)$ that yields the following result.

Corollary 2. The threshold of the optimal contract is given by

$$
\bar{\theta}=\frac{1-F(\bar{\theta})}{f(\bar{\theta})} .
$$


This result implies that among high valuation agents there always exist some types that are exploited and some that are not since the optimal threshold can never be 1 . In other words, the expected profit of the principal is greater under the present model than it is under any conventional price discrimination model where no agent's bounded rationality is exploited. That is, the benefit of discrimination based on cognitive limitations to the principal is always higher that its cost. What this cost and benefit are will be stated properly in the following subsection.

Now let us turn our attention to the menu designed for relatively sophisticated high valuation types. According to Proposition 3, the primary and the secondary product of this menu provide the same utility for high valuation types. Since only the primary products affect the principal's profit, without loss of generality, the secondary product of this menu could be considered to be the same as its primary product.

Proposition 6. The product designed for high valuation types with $\theta \leq \bar{\theta}$ is such that $v^{\prime}\left(q_{0}\right)=c^{\prime}\left(q_{0}\right)$.

As in the menu designed for naïve high valuation types, the quality of the product is such that its marginal cost is equal to its marginal utility. This implies that the principal uses the same quality product in both menus and exploits naïve types by charging them more. Even though they are gravitated towards the menu either by an expensive and high quality or by a cheap and low quality product, naïve types end up buying the same (quality) product as sophisticated types but pay more. This observation provides a possible explanation to the apparent puzzle why one may observe products of the same quality sold at different prices under different labels.

The following proposition describes the menu designed for low valuation types and thus completes the characterization of the optimal contract.

Proposition 7. The products designed for low valuation types are such that

$$
c^{\prime}\left(q_{l}\right)=v_{l}^{\prime}\left(q_{l}\right)-\frac{1-\lambda}{\lambda}\left[v^{\prime}\left(q_{l}\right)-v_{l}^{\prime}\left(q_{l}\right)\right][1-\bar{\theta}+\bar{\theta} F(\bar{\theta})]
$$

where $\bar{\theta}=\frac{1-F(\bar{\theta})}{f(\bar{\theta})}$.

The incentive to choose this menu is not given by exploiting the types' cognitive limitation but through their preferences. The products in both menus designed for high valuation types cost more than what low valuation types are willing to pay. Therefore, they choose the menu whose products cost exactly the same as their willingness to pay and extract their entire surplus. Without loss of generality, the primary and secondary products of this menu could be considered to be the same, since they both provide these types with the same utility.

Proposition 7 shows that the quality of the product chosen by low valuation types is lower than its value under the perfect information, but higher than it is under 
any conventional price discrimination model. In asymmetric information case, the principal cannot escape from paying informational rents. This causes him to lower the quality of the product designed for low valuation consumers relative to full information case. However, when the principal takes into account that agents are diversely context biased, he can exploit their bounded rationality and decrease the informational rents he is paying. The difference in the quality level between the present and an ordinary price discrimination model can be observed from Equation 7. This equation is the same as in any ordinary price discrimination model except for the last term, $1-\theta+\theta F(\theta)$. This additional term is less than 1 for all values of $\theta \in[0,1]$, and therefore, it increases the quality level by decreasing the informational rents. Furthermore, the optimal threshold $\bar{\theta}$, given in Corollary 2 , minimizes this additional term. That is, the optimal contract maximizes the profit by minimizing informational rents paid to high valuation types.

Propositions 5, 6 and 7 characterize the optimal contract entirely and imply that

i. low valuation types are designed a menu that consists of a cheap and low quality product,

ii. relatively sophisticated high valuation types are given informational rents in order to prevent them from mimicking low valuation types. Furthermore, the comparison between Proposition 6 and 7 implies $q_{0}>q_{l}$, since the cost function $c($.$) is$ convex. That is, the product designed for sophisticated high valuation types has a higher quality than the one designed for low valuation types.

iii. relatively naïve high valuation types are the only ones whose bounded rationality is exploited. They are provided with a product whose quality is the same as the one sold to sophisticated high valuation types but are charged more.

\section{The decision to discriminate}

According to Corollary 2 in the previous section, the optimal threshold, $\bar{\theta}$ is strictly less than 1. This implies that the principal's expected profit is strictly greater under the present model than it is under a standard price discrimination model (henceforth, the benchmark model). That is, the cost of discriminating agents based on their cognitive limitation, if any, is less than its benefit. This section studies these cost and benefit by taking a closer look at the differences between the present and the benchmark model.

The following observation presents the characteristics of the contract under the benchmark model.

Observation 2. The optimal contract under the benchmark model has two menus, each of which contains only a single product. Low valuation types are sold a product $\left(q_{l}^{b}, p_{l}^{b}\right)$ such that 


$$
\begin{aligned}
c^{\prime}\left(q_{l}^{b}\right) & =v_{l}^{\prime}\left(q_{l}^{b}\right)-\frac{1-\lambda}{\lambda}\left[v_{h}^{\prime}\left(q_{l}^{b}\right)-v_{l}^{\prime}\left(q_{l}^{b}\right)\right] \text { and } \\
p_{l}^{b} & =v_{l}\left(q_{l}^{b}\right)
\end{aligned}
$$

whereas the product designed for high valuation types, $\left(q_{h}^{b}, p_{h}^{b}\right)$, is such that

$$
\begin{aligned}
c^{\prime}\left(q_{h}^{b}\right) & =v_{h}^{\prime}\left(q_{h}^{b}\right) \text { and } \\
p_{h}^{b} & =v_{h}\left(q_{h}^{b}\right)-\left[v_{h}\left(q_{l}^{b}\right)-v_{l}\left(q_{l}^{b}\right)\right],
\end{aligned}
$$

where the superscript $b$ stands for the benchmark.

There are two main differences between the two contracts: the quality of the product designed for low valuation types and the price charged from naïve high valuation types. As noted in the previous section, low valuation types are provided with a higher quality product under the present model, $q_{l}>q_{l}^{b}$. The facts that (i) the principal's objective function is strictly concave, (ii) $q_{l}$ is greater than $q_{l}^{b}$ but smaller than its value under the perfect information case and (iii) the entire surplus is extracted from low valuation types in both models lead to the conclusion that the principal's profit from these types is strictly higher under the present model. That is, the first benefit of discriminating agents based on their cognitive limitation is due to the increase in profit coming from low valuation types. Another implication of the difference between $q_{l}$ and $q_{l}^{b}$ is that the price charged from sophisticated high valuation types is lower under the present model. The intuition behind this is as follows. Low valuation types are provided with a higher quality product but in order to extract their entire surplus they are also charged more. That is, the increase in quality is accompanied with an increase in price so that the overall change does not affect the utility of a low valuation type. However this change increases the utility that a high valuation type gets from this product. In order to give her the incentive to choose the menu designed for her own type, the informational rents she receives must be increased. This is done by decreasing the price she is charged. That is, the second effect of the increase in $q_{l}$ is that it decreases the principal's profit by causing a decrease in the price of the product designed for sophisticated high types. Furthermore, naïve high valuation types also receive informational rents but only partially. Discriminating agents based on their cognitive limitation has two opponent effects on the profit extracted from these types. On the one hand, the profit decreases due to the increase in the informational rents these types receive. This is exactly the same effect that is observed with sophisticated high valuation types. But on the other hand, it increases due to the fact that these types' cognitive limitations are exploited making use of attention grabbers and more is extracted from them. Putting all the pieces together, the total cost of discriminating agents based on their cognitive limitation is due to the increase in informational rents high valuation types receive. 
And it is equal to $(1-\lambda)\left[\Delta\left(q_{l}\right)-\Delta\left(q_{l}^{b}\right)\right]$, where $\Delta v(q)=v_{h}(q)-v_{l}(q)$. The benefit, instead, is $\lambda\left[v_{l}\left(q_{l}\right)-c\left(q_{l}\right)-v_{l}\left(q_{l}^{b}\right)+c\left(q_{l}^{b}\right)\right]+(1-\lambda)(1-F(\bar{\theta})) \bar{\theta} \Delta\left(q_{l}\right)$ and it comes from the facts that the quality of the product sold to low valuation types is closer to its first-best level and naïve high valuation types are exploited.

The difference in principal's expected profit between the two contracts can be written as the difference between benefit and cost. That is,

$$
\Delta \Pi=\lambda\left[\left(v_{l}\left(q_{l}\right)-c\left(q_{l}\right)\right)-\left(v_{l}\left(q_{l}^{b}\right)-c\left(q_{l}^{b}\right)\right)\right]+(1-\lambda)\left[\Delta v\left(q_{l}^{b}\right)-(1-\bar{\theta}+\bar{\theta} F(\bar{\theta})) \Delta v\left(q_{l}\right)\right] .
$$

This difference is a function of the threshold, $\bar{\theta}$, since it simultaneously determines the fraction of high valuation types who are exploited and the amount of this exploitation. The value of this function is zero at both extremes, i.e., $\Delta \Pi(0)=\Delta \Pi(1)=0$. Furthermore, the facts that (i) its right-hand limit at 0 is positive, (ii) the left-hand limit at 1 is positive and (iii) the only stationary point is at $\bar{\theta}=\frac{1-F(\bar{\theta})}{f(\bar{\theta})}$ imply that this stationary point is the maximum. This result confirms the optimality of the threshold given in Corollary 2 by showing that it maximizes the extra profit that the principal collects when discriminating agents based on cognitive limitations.

\section{Welfare}

This section considers the welfare difference compared to the benchmark model and shows that the contract discriminating agents based on cognitive imperfections is welfare improving.

As shown in Section 4, this contract partitions the type space of agents into three subsets as low valuation, sophisticated high valuation and naïve high valuation types. Low valuation types end up with zero utility in the present, as well as in the benchmark model. Thus, there is no difference in the welfare of these types. The cost of discriminating agents based on cognitive limitation, borne by the principal, comes from the increase in the informational rents he pays. Therefore, this is reflected as benefit to high valuation types. As a result, these types' welfare increases by $\Delta\left(q_{l}\right)-\Delta\left(q_{l}^{b}\right){ }^{4}$. For naïve high valuation types, however, this is not the only effect of this contract. Since it exploits these types' cognitive imperfections, their utility decreases by $\bar{\theta} \Delta\left(q_{l}\right)$. This is the amount of benefit that the principal captures from these naïve types. Hence, the overall change in the welfare of high valuation types is

$$
(1-\bar{\theta}+\bar{\theta} F(\bar{\theta})) \Delta v\left(q_{l}\right)-\Delta v\left(q_{l}^{b}\right) .
$$

In fact, this is the total welfare difference between two models on agents' side since there is no change in low valuation types' utility.

\footnotetext{
${ }^{4}$ See Section 5 for the cost and benefit of the principal.
} 
As noted earlier, the costs (benefits) of the principal from high valuation types are reflected as gains (losses) on these types. Therefore, they have zero net effect on the aggregate welfare. However, this is not the case for low valuation types. The principal's profit from these types increases since the quality of the product designed for them is closer to its value under the full information case. But this does not make any change in their welfare since their utility remains the same as in the benchmark model. This implies that the benefits extracted from low valuation types do increase the aggregate welfare by $\lambda\left[\left(v_{l}\left(q_{l}\right)-c\left(q_{l}\right)\right)-\left(v_{l}\left(q_{l}^{b}\right)-c\left(q_{l}^{b}\right)\right)\right]$. Hence, discriminating agents based on their cognitive limitations improves the welfare.

\section{Conclusions}

This paper deals with a sort of cognitive limitation that may lead individuals to make context dependent decisions. Specifically, it considers the fact that introducing an inferior option into a category may increase its attractiveness and cause agents to gravitate towards it. Integrating this type of behavior into mechanism design, this paper characterizes a principal's optimal menu of contracts when agents differ both in preferences and in the level of cognitive limitations. That is, the principal is facing a situation in which a type in the market is a two dimensional object. The first component represents her valuation for quality which is a binary variable taking values high or low. The second component, denoted by $\theta$, is the level of cognitive limitation which takes its values in a continuum.

In conventional price discrimination models, the entire surplus is extracted from low valuation types. High valuation types, instead, are given informational rents in order to be prevented from mimicking low valuation types. This paper shows that the principal can design a mechanism that screens high valuation types' degree of sophistication and exploits their bounded rationality. The optimal contract, in this case, has three menus. The first one is designed for low valuation types and, as in conventional models, extracts their entire surplus. The last two menus partition the space of high valuation types into two subsets based on a threshold level for $\theta$. Due to their sophistication, the principal cannot extract much from high valuation types who fall below the threshold. That is, relatively sophisticated high valuation types' bounded rationality is not exploited and therefore, they receive full informational rents. The principal exploits the rest of high valuation types and extracts more from them. Thus, these types receive only partial informational rents. A change in the threshold has two opposing effects. The higher the $\theta$, the more naïve the types are. Therefore, increasing the threshold means that the most sophisticated type in the set of exploited types is more naïve. Hence, the principal can extract a higher amount. On the other side, it causes a loss due to shrinkage in the set of exploited types. In other words, increasing the threshold, the principal can extract a higher amount but from a smaller 
subset of agents. At optimum, the threshold is such that the benefit from a marginal change is offset by the loss it creates.

The optimal contract offers the same quality products in both menus designed for high valuation types but with different prices. Naïve types end up paying more for the same quality product sold to more sophisticated types. This observation provides a possible explanation to the apparent puzzle why one may observe products of the same quality sold at different prices under different labels. Furthermore, low valuation types are sold a product that has a lower quality than the one sold to high valuation types. The conclusions suggest that a seller manufacturing jeans, for example, should create three different brands. The first one is for low valuation agents and the jeans under this brand are cheap but of low quality. The second brand is for sophisticated high valuation agents and high quality jeans are sold with reasonable prices. Finally, the third one is a premium brand and is designed for naïve high valuation agents. Under this label, the same high quality jeans as in the second brand are sold with high prices.

Regarding high valuation types, benefits that the principal captures by discriminating them based on cognitive imperfections are reflected as losses on these types, and similarly, costs borne by the principal as gains. However, this is not the case with low valuation types. Even though the principal's profit obtained from these types is strictly higher when he takes agents' cognitive limitations into account, it makes no difference in their welfare. Like in any conventional price discrimination model, their entire surplus is extracted. Therefore, discriminating agents based on their cognitive limitations improves the aggregate welfare. 


\section{A Appendix}

Proof of Proposition 1. We start this proof by ignoring the incentive compatibility constraints of low valuation types, and later on we will show that the results obtained satisfy these constraints. Now, assume that the optimal contract includes a menu designed for some low valuation types that does not extract their entire surplus. Call this menu as menu $A$ and denote its primary and secondary products by $\left(q_{a}, p_{a}\right)$ and $\left(q_{a}^{\prime}, p_{a}^{\prime}\right)$, respectively. Since this menu does not extract the entire surplus, its primary product provides strictly positive utility to low valuation types, $u_{l}\left(q_{a}, p_{a}\right)>0$. If the utility provided by the secondary product of this menu is strictly lower than $u_{l}\left(q_{a}, p_{a}\right)$, then take an $\epsilon$ such that $u_{l}\left(q_{a}, p_{a}\right)-u_{l}\left(q_{a}^{\prime}, p_{a}^{\prime}\right) \geq \epsilon>0$ and consider the adjustment that increases the price of the primary product: $p_{a}^{n e w}=p_{a}+\epsilon$. If, instead, the utility provided by the secondary product of this menu is exactly $u_{l}\left(q_{a}, p_{a}\right)$, then take an $\epsilon$ such that $u_{l}\left(q_{a}, p_{a}\right) \geq \epsilon>0$ and consider the adjustment that increases the price of both the primary and the secondary products: $p_{a}^{\text {new }}=p_{a}+\epsilon$ and $p_{a}^{\text {new }}=p_{a}^{\prime}+\epsilon$. In both cases, the primary product provides higher utility than the secondary product and both utility levels are non-negative for a low valuation type. That is, $u_{l}\left(q_{a}, p_{a}^{\text {new }}\right) \geq u_{l}\left(q_{a}^{\prime}, p_{a}^{\prime \text { new }}\right) \geq 0$. Furthermore, this adjustment relaxes the incentive compatibility constraints of high valuation types by decreasing their valuation for menu $A$. Therefore it does not violate any constraints of the principal's optimization problem while increasing his profit. This results contradicts with the optimality of the contract and implies that there exists only a single menu that extracts the entire surplus from low valuation types.

It remains to check that the resulting contract does not give low valuation types an incentive to choose any menu designed for high valuation types. In order to accomplish this goal, we need to jump forward and use the results from Proposition 5 and Proposition 6. First, we consider the menu designed for sophisticated high valuation types. The utility that a low valuation type gets from the primary and the secondary product of this menu is $v_{l}\left(q_{0}\right)-v_{h}\left(q_{0}\right)+v_{h}\left(q_{l}\right)-v_{l}\left(q_{l}\right)$ and it is strictly negative since $q_{0}>q_{l}$ and the single crossing property holds. Therefore his valuation for this menu is zero. Now, consider the menu designed for naive high valuation types. The utility that a low valuation type gets from the primary and the secondary product of this menu are $v_{l}\left(q_{\theta}\right)-v_{h}\left(q_{\theta}\right)+(1-\bar{\theta})\left(v_{h}\left(q_{l}\right)-v_{l}\left(q_{l}\right)\right)$ and $v_{l}\left(q_{\theta}^{\prime}\right)-v_{h}\left(q_{\theta}^{\prime}\right)$, respectively. These utilities are both strictly negative and hence his valuation for this menu is zero as well.

Proof of Proposition 2. Consider two high valuation types whose level of context-bias are $\theta_{1}$ and $\theta_{2}$. Assume $\theta_{1}<\theta_{2}$ and these types choose different menus of the contract, say $A$ and $B$. Denote the primary and secondary products of menu $A$ as $\left(a, a^{\prime}\right)$ and menu $B$ as $\left(b, b^{\prime}\right)$. From the incentive compatibility constraints for these two type we 
get

$$
\begin{aligned}
& u(a)-\theta_{1} u\left(a^{\prime}\right) \geq u(b)-\theta_{1} u\left(b^{\prime}\right) \\
& u(b)-\theta_{2} u\left(b^{\prime}\right) \geq u(a)-\theta_{2} u\left(a^{\prime}\right)
\end{aligned}
$$

Combining these two inequalities yields $\theta_{2}\left(u\left(a^{\prime}\right)-u\left(b^{\prime}\right)\right) \geq u(a)-u(b) \geq \theta_{1}\left(u\left(a^{\prime}\right)-u\left(b^{\prime}\right)\right)$. Since $\theta_{1}<\theta_{2}$, we conclude that $u\left(a^{\prime}\right) \geq u\left(b^{\prime}\right)$ and $u(a) \geq u(b)$.

Proof of Corollary 1. Suppose that a menu, say A, whose primary and secondary products are denoted by $\left(a, a^{\prime}\right)$ is chosen by two different high valuation types whose levels of context bias are $\theta_{1}$ and $\theta_{2}$. Assume that there exists a high valuation $\theta_{3}$-type, where $\theta_{3} \in\left(\theta_{1}, \theta_{2}\right)$, and that this type choses another menu, say B. From incentive compatibility constraints, we have

$$
\begin{aligned}
& u(a)-\theta_{1} u\left(a^{\prime}\right) \geq u(b)-\theta_{1} u\left(b^{\prime}\right) \\
& u(a)-\theta_{2} u\left(a^{\prime}\right) \geq u(b)-\theta_{2} u\left(b^{\prime}\right) \\
& u(b)-\theta_{3} u\left(b^{\prime}\right) \geq u(a)-\theta_{3} u\left(a^{\prime}\right)
\end{aligned}
$$

If $u\left(a^{\prime}\right) \geq u\left(b^{\prime}\right)$, these three inequalities imply

$$
\theta_{3}\left(u\left(a^{\prime}\right)-u\left(b^{\prime}\right)\right) \geq u(a)-u(b) \geq \theta_{2}\left(u\left(a^{\prime}\right)-u\left(b^{\prime}\right)\right) \geq \theta_{1}\left(u\left(a^{\prime}\right)-u\left(b^{\prime}\right)\right),
$$

and due to the fact that $0 \leq \theta_{1}<\theta_{3}<\theta_{2}$ we have $u(a)=u(b)$ and $u\left(a^{\prime}\right)=u\left(b^{\prime}\right)$. If , instead, $u\left(a^{\prime}\right) \leq u\left(b^{\prime}\right)$, the three inequalities imply

$$
\theta_{3}\left(u\left(a^{\prime}\right)-u\left(b^{\prime}\right)\right) \geq u(a)-u(b) \geq \theta_{1}\left(u\left(a^{\prime}\right)-u\left(b^{\prime}\right)\right) \geq \theta_{2}\left(u\left(a^{\prime}\right)-u\left(b^{\prime}\right)\right),
$$

and we again have $u(a)=u(b)$ and $u\left(a^{\prime}\right)=u\left(b^{\prime}\right)$. Therefore, menu $A$ is the same as menu $B$.

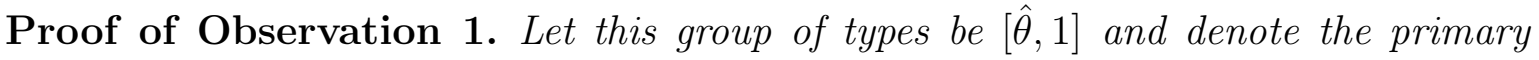
and the secondary products of the menu they choose as a and $a^{\prime}$, respectively. Assume $u\left(a^{\prime}\right)>0$. Take an $\epsilon$ such that $0<\epsilon \leq u\left(a^{\prime}\right)$ and consider the following adjustment:

$$
\begin{aligned}
& p^{n e w}(a)=p(a)+\hat{\theta} \epsilon \\
& p^{n e w}\left(a^{\prime}\right)=p\left(a^{\prime}\right)+\epsilon
\end{aligned}
$$

This adjustment decreases utilities provided by the primary and the secondary product by increasing their prices. It makes the menu more attractive for high valuation types whose level of context-bias belongs to $[\hat{\theta}, 1]$, whereas the menu becomes less attractive for all other types. Thus, this adjustment does not violate any constraints of the principal's problem while increasing his profit. Therefore we have a contradiction. 
Proof of Proposition 3. Let us denote the primary and the secondary products of the menu designed for the partition block of the type space to which the fully rational high valuation type belongs by $a$ and $a^{\prime}$, respectively. The fully rational type $(\theta=0)$ does not fall for the attraction of attention grabbers and considers only the primary products of menus when evaluating them. Therefore, he never chooses an exploitative menu. Incentive compatibility constraints imply that his valuation of the menu designed for him should be at least the same as his valuation of the menu designed for low valuation types. In fact this incentive compatibility constraint binds, since otherwise the principal can make a profitable adjustment by increasing the price he charges from this type. Thus we have $u(a)=u\left(q_{l}, p_{l}\right)$.

Now assume that the secondary product of this menu provides a strictly lower utility than the primary product, that is $u\left(a^{\prime}\right)<u(a)$. Take an $\epsilon$ such that $0<\epsilon \leq u(a)-u\left(a^{\prime}\right)$ and consider the following adjustments:

$$
\begin{aligned}
& \text { i. } p\left(a^{\prime}\right)^{\text {new }}=p\left(a^{\prime}\right)-\epsilon \\
& \text { ii. } q_{l}^{\prime \text { new }}=q_{l}^{\prime}+x \text {, where }\left[v_{h}\left(q_{l}^{\prime}+x\right)-v_{l}\left(q_{l}^{\prime}+x\right)\right]-\left[v_{h}\left(q_{l}^{\prime}\right)-v_{l}\left(q_{l}^{\prime}\right)\right]=\epsilon \\
& \text { iii. } p_{l}^{\prime \text { new }}=p_{l}^{\prime}+\left[v_{l}\left(q_{l}^{\prime}+x\right)-v_{l}\left(q_{l}^{\prime}\right)\right] \\
& \text { iv. } p_{\theta}^{\text {new }}=p_{\theta}+\bar{\theta} \epsilon \text { for all the menus designed for the rest of high valuation types. }
\end{aligned}
$$

The first adjustment increases the utility received from the secondary product $a^{\prime}$, thus decreases the attractiveness of the menu. The second adjustment together with the third one imply that both the price and the quality of the secondary product for low valuation types are increased such that the utility of a low valuation type from this product is not changed, whereas the utility of a high valuation type is increased by $\epsilon$. This implies that the attractiveness of the menu designed for low valuation types decreases only for high valuation types. The last adjustment increases the prices of primary products in all other menus designed for high valuation types. Types with $\theta \in[0, \bar{\theta})$ continue to choose the menu designed for their type, since the decrease in their valuation for this menu is less than the decrease for other menus. Furthermore, these adjustments do not cause also the rest of high valuation types to change the menu they choose. Their subjective valuations for the menu designed for high valuation types with $\theta \in[0, \bar{\theta})$ and the menu designed for low valuation types decrease at least by $\bar{\theta} \epsilon$, whereas for all the other menus it decreases by exactly $\bar{\theta} \epsilon$. Since the principal's profit increases after these adjustments that do not violate any constraints of the problem, we have $u\left(a^{\prime}\right)=u(a)$ in the optimal contract.

Proof of Proposition 4. We start the proof by ignoring the incentive compatibility constraints of high valuation types related to the menu designed for low valuation types and then finalize it by showing that the result obtained satisfies them as well. 
Observe that incentive compatibility constraint for high valuation types can be written as follows:

$$
V(\theta)=\max _{\phi \in[0,1]}\left\{u\left(q_{\phi}, p_{\phi}\right)-\theta u\left(q_{\phi}^{\prime}, p_{\phi}^{\prime}\right)\right\} .
$$

Furthermore, $u\left(q_{\phi}, p_{\phi}\right)-\theta u\left(q_{\phi}^{\prime}, p_{\phi}^{\prime}\right)$ is an affine function of $\theta$, since $u\left(q_{\phi}^{\prime}, p_{\phi}^{\prime}\right) \geq 0$ for each $\phi \in[0,1]$. Being a maximum of a family of affine functions, $V(\theta)$ is convex and therefore it is absolutely continuous and differentiable almost everywhere. Furthermore, for each $\theta \in[0,1]$ we have

$$
V(\phi) \geq u\left(q_{\theta}, p_{\theta}\right)-\phi u\left(q_{\theta}^{\prime}, p_{\theta}^{\prime}\right)=V(\theta)+u\left(q_{\theta}^{\prime}, p_{\theta}^{\prime}\right)(\theta-\phi) .
$$

This implies that $V^{\prime}(\theta)=-u\left(q_{\theta}^{\prime}, p_{\theta}^{\prime}\right)$ and using the first fundamental rule of calculus we obtain

$$
V(\theta)=V(0)-\int_{0}^{\theta} u\left(q_{x}^{\prime}, p_{x}^{\prime}\right) \mathrm{d} x=u\left(q_{0}, p_{0}\right)-\int_{0}^{\theta} u\left(q_{x}^{\prime}, p_{x}^{\prime}\right) \mathrm{d} x
$$

Now, it remains to check that the expression derived above satisfies the incentive compatibility constraints of high valuation types related to the menu designed for low valuation types. That is, we need to show

$$
V(\theta)=u\left(q_{0}, p_{0}\right)-\int_{0}^{\theta} u\left(q_{x}^{\prime}, p_{x}^{\prime}\right) \mathrm{d} x \geq u\left(q_{l}, p_{l}\right)-\theta u\left(q_{l}^{\prime}, p_{l}^{\prime}\right)
$$

for any $\theta \in[0,1]$. This follows from the facts that $u\left(q_{0}, p_{0}\right)=u\left(q_{0}^{\prime}, p_{0}^{\prime}\right)=u\left(q_{l}, p_{l}\right)=$ $u\left(q_{l}^{\prime}, p_{l}^{\prime}\right)$ and $u\left(q_{\theta}^{\prime}, p_{\theta}^{\prime}\right)$ is decreasing in $\theta$, which are obtained in Propositions 3 and 2 , respectively.

Proof of Remark 1. Consider the last term of the objective function which can be expressed as follows:

$$
\begin{array}{r}
(1-\lambda) \int_{\bar{\theta}}^{1}\left[v\left(q_{\theta}\right)-c\left(q_{\theta}\right)-\theta u\left(q_{\theta}^{\prime}, p_{\theta}^{\prime}\right)-(1-\bar{\theta})\left(v\left(q_{l}\right)-v_{l}\left(q_{l}\right)\right)\right] f(\theta) \mathrm{d} \theta \\
+(1-\lambda) \int_{\bar{\theta}}^{1} \int_{\bar{\theta}}^{\theta} u\left(q_{x}^{\prime}, p_{x}^{\prime}\right) f(\theta) \mathrm{d} x \mathrm{~d} \theta
\end{array}
$$

Changing the order of integration the second term becomes

$$
(1-\lambda) \int_{\bar{\theta}}^{1} \int_{x}^{1} u\left(q_{x}^{\prime}, p_{x}^{\prime}\right) f(\theta) \mathrm{d} \theta \mathrm{d} x=(1-\lambda) \int_{\bar{\theta}}^{1} u\left(q_{x}^{\prime}, p_{x}^{\prime}\right)(1-F(x)) \mathrm{d} x
$$


Now substituting this term back into (8) we get

$$
\begin{aligned}
& =(1-\lambda) \int_{\bar{\theta}}^{1}\left\{v\left(q_{\theta}\right)-c\left(q_{\theta}\right)-(1-\bar{\theta})\left(v\left(q_{l}\right)-v_{l}\left(q_{l}\right)\right)+u\left(q_{\theta}^{\prime}, p_{\theta}^{\prime}\right)\left[\frac{1-F(\theta)}{f(\theta)}-\theta\right]\right\} f(\theta) \mathrm{d} \theta \\
& =(1-\lambda) \int_{\bar{\theta}}^{1}\left\{v\left(q_{\theta}\right)-c\left(q_{\theta}\right)-(1-\bar{\theta})\left(v\left(q_{l}\right)-v_{l}\left(q_{l}\right)\right)-\left(v\left(q_{\theta}^{\prime}\right)-p_{\theta}^{\prime}\right) \Phi(\theta)\right\} f(\theta) \mathrm{d} \theta,
\end{aligned}
$$

where $\Phi(\theta)=\theta-\frac{1-F(\theta)}{f(\theta)}$.

Before continuing with the proofs of the remaining propositions that characterize the optimal contract, we restate the principal's optimization problem in its final form.

Observation 3. The principal's optimization problem can be stated as follows:

$$
\begin{aligned}
\max _{\left\{q_{l}, q_{0}, q_{\theta},\left(q_{\theta}^{\prime}, p_{\theta}^{\prime}\right)\right\}} \lambda\left[v_{l}\left(q_{l}\right)-c\left(q_{l}\right)\right]+(1-\lambda) F(\bar{\theta})\left[v\left(q_{0}\right)-v\left(q_{l}\right)+v_{l}\left(q_{l}\right)-c\left(q_{0}\right)\right] \\
+(1-\lambda) \int_{\bar{\theta}}^{1}\left[v\left(q_{\theta}\right)-c\left(q_{\theta}\right)-(1-\bar{\theta})\left(v\left(q_{l}\right)-v_{l}\left(q_{l}\right)\right)-\Phi(\theta)\left(v\left(q_{\theta}^{\prime}\right)-p_{\theta}^{\prime}\right)\right] f(\theta) \mathrm{d} \theta
\end{aligned}
$$

subject to $\quad u\left(q_{\theta}^{\prime}, p_{\theta}^{\prime}\right) \geq 0$

Proofs of Propositions 5, 6 and 7. We can solve the problem given in (9) by point-by-point optimization. The first order conditions with respect to $q_{\theta}$ and $\left(q_{\theta}^{\prime}, p_{\theta}^{\prime}\right)$ are

$$
v^{\prime}\left(q_{\theta}\right)=c^{\prime}\left(q_{\theta}\right)
$$

and

$$
\begin{cases}v\left(q_{\theta}^{\prime}\right)=p_{\theta}^{\prime} & \text { if } \Phi(\theta)>0 \\ v\left(q_{\theta}^{\prime}\right)-p_{\theta}^{\prime}=v\left(q_{\theta}\right)-p_{\theta} & \text { if } \Phi(\theta)<0\end{cases}
$$

From the first order conditions given in (10), we see that the secondary product designed for types for whom $\Phi(\theta)$ is less than zero provides the same utility as their primary product, that is, $u\left(q_{\theta}, p_{\theta}\right)=u\left(q_{\theta}^{\prime}, p_{\theta}^{\prime}\right)$. However, this menu is the same as the one designed for sophisticated high valuation types (see Proposition 3). This implies that the types for whom $\Phi(\theta)$ is less than zero must be included to the block of type space partition for which the non-exploitative menu is designed. In all exploitative menus, we have high valuation types for whom $\Phi(\theta)$ is greater than zero and therefore $v\left(q_{\theta}^{\prime}\right)=p_{\theta}^{\prime}$.

The first order conditions with respect to $q_{0}$ and $q_{l}$ give

$$
v^{\prime}\left(q_{0}\right)=c^{\prime}\left(q_{0}\right)
$$


and

$$
c^{\prime}\left(q_{l}\right)=v_{l}^{\prime}\left(q_{l}\right)-\frac{1-\lambda}{\lambda}\left[v^{\prime}\left(q_{l}\right)-v_{l}^{\prime}\left(q_{l}\right)\right][1-\bar{\theta}+\bar{\theta} F(\bar{\theta})],
$$

respectively.

Proof of Remark 2. Assume, on the contrary, that there exist more than one menu designed for high valuation agents with $\theta \geq \bar{\theta}$. Consider two such menus; $A$ and $B$, and denote their primary and secondary products by $\left(a, a^{\prime}\right)$ and $\left(b, b^{\prime}\right)$, respectively. From Proposition 5 we know that the secondary products of both menus provide zero utility for high valuation types, that is $u\left(a^{\prime}\right)=u\left(b^{\prime}\right)=0$. A type who chooses menu $A$ does not deviate to menu $B$ as long as he has higher subjective valuation for menu $A, u(a) \geq u(b)$. Likewise, for the incentive compatibility constraint of a type choosing menu $B$ to be satisfied, we need $u(b) \geq u(a)$. This implies $u(a)=u(b)$. The fact that both the primary and the secondary products of menu $A$ and $B$ provide the same utilities implies that these two menus are the same.

Proof of Corollary 2. In the proof of Proposition 5, we have seen that for all $\theta$ such that $\Phi(\theta)<0$ primary and secondary products provide the same utility and for all $\theta$ such that $\Phi(\theta)>0$ secondary products serve as attention grabbers and provide zero utility, see equation 10. This implies that the optimal threshold $\bar{\theta}$ is such that $\Phi(\bar{\theta})=0$, which implies $\bar{\theta}=\frac{1-F(\bar{\theta})}{f(\bar{\theta})}$. 


\section{References}

[1] Barbos, A. (2010), "Context Effects: A Representation of Choices from Categories", Journal of Economic Theory 145 1224-1243.

[2] Doyle, J. R., O'Connor, D. J., Reynolds, G. M., and Bottomley, P. A. (1999), "The robustness of the asymmetrically dominated effect: Buying frames, phantom alternatives, and in-store purchases", Psychology $\&$ Marketing, 16(3), 225-243.

[3] Eliaz, K., and R. Spiegler (2006), "Contracting with Diversely naïve Agents", Review of Economic Studies, 73(3), 689-714.

[4] Eliaz, K., R. Spiegler (2010), "On the Strategic Use of Attention Grabbers", Theoretical Economics, 6(1), 127-155.

[5] Huber, Payne and Puto (1982), "Adding asymmetrically dominated alternatives: Violations of regularity and the similarity hypothesis", Journal of consumer research, 90-98.

[6] Piccione, M. and Rubinstein, A. (2003), "Modeling the Economic Interaction of Agents with Diverse Abilities to Recognize Equilibrium Patterns", Journal of European Economic Association, 1, 212-223.

[7] Rubinstein, A. (1993), "On Price Recognition and Computational Complexity in a Monopolistic Model", Journal of Political Economy, 101, 473-484.

[8] Simonson, I. and Tversky, A. (1992), "Choice in context: Trade-off Contrast and Extremeness Aversion", J. Marketing Res., 29, 281-295.

[9] Tversky, A., and D. Kahneman (1981). "The Framing of Decision and the Psychology of Choice," Science, 211, 453-458. 